\title{
Perioperative Echocardiographic Diagnosis of Regional Wall Motion Abnormalities: Not All of Them are Ischemic!
}

\author{
${ }^{1}$ Michael L Boisen, ${ }^{2}$ Stephen M McHugh, ${ }^{3}$ Robert H Boretsky, ${ }^{4}$ Dennis P Phillips, ${ }^{5}$ Li Meng \\ ${ }^{6}$ Mathew W Caldwell, ${ }^{7} \mathrm{~A}$ Murat Kaynar, ${ }^{8}$ Kathirvel Subramaniam
}

\begin{abstract}
We present three noncardiac surgical patients with regional left ventricular dysfunction diagnosed by bedside echocardiography: One intraoperative, another immediate postoperative, and a third one with preoperative hemodynamic instability. We review the differential diagnosis and the growing role of perioperative transthoracic echocardiography.
\end{abstract}

Keywords: Ischemia, Perioperative, Transthoracic echocardiography, Wall motion.

How to cite this article: Boisen ML, McHugh SM, Boretsky RH, Phillips DP, Meng L, Caldwell MW, Kaynar AM, Subramaniam K. Perioperative Echocardiographic Diagnosis of Regional Wall Motion Abnormalities: Not All of Them are Ischemic! J Perioper Echocardiogr 2016;4(2):70-73.

Source of support: Nil

Conflict of interest: None

\section{INTRODUCTION}

Regional wall motion abnormalities (RWMA) are frequently encountered by echo cardiographers during evaluation for hemodynamic instability. It is important to differentiate true wall motion changes induced by ischemia from other causes of RWMA. Ischemic WMA will be confined to the coronary artery territory unlike non-ischemic WMA. Other reasons for WMA such as arrhythmias, hypovolemia and increased afterload should be identified and treated. Stress induced cardiomyopathy is another reason for WMA but coronary artery distribution is absent and cardiac catheterization will show no significant disease. Treatment is supportive and this condition should be recognized during the stressful perioperative period of non-cardiac surgical procedures. Here we describe three patients with the diagnosis of stress induced cardiomyopathy evaluated by preoperative transesophageal and transthoracic echocardiography and present their clinical course.

$1,2,4,6$ Clinical Assistant Professor, ${ }^{3}$ Clinical Associate Professor ${ }^{5,7,8}$ Associate Professor

${ }^{1-8}$ Department of Anesthesiology, University of Pittsburgh Medical Center, Pittsburgh, Pennsylvania, USA

Corresponding Author: Kathirvel Subramaniam, Associate Professor, Department of Anesthesiology, University of Pittsburgh Medical Center, Pittsburgh, Pennsylvania, USA Phone: +004126475635, e-mail: subramaniamk@upmc.edu

\section{CASE REPORTS}

\section{Case 1}

A 68-year-old female with history of chronic pain, anemia, hyperlipidemia, hypertension, and scoliosis presented for T3 to pelvis posterior spinal fusion. Medications included lisinopril, propranolol, clonazepam, sertraline, and quetiapine. She had no cardiac history and functional status was approximately 4 metabolic equivalents; therefore, no cardiac testing beyond chest radiograph and electrocardiogram (ECG) was done preoperatively. After uneventful induction of general anesthesia and placement of arterial and central venous catheters, surgery proceeded. During spine instrumentation, severe hypotension (systolic blood pressure $40 \mathrm{~mm} \mathrm{Hg}$ ) occurred refractory to crystalloid and colloid infusion, blood transfusion, and phenylephrine and epinephrine and norepinephrine were required to support blood pressure. Following this episode, blood pressure was elevated and diffuse ST-segment elevations were noted, which normalized after hypertension was controlled. Total blood loss was estimated at 1.5 L. At the conclusion of surgery, the patient was returned to supine position and transthoracic echocardiography (TTE) performed by a cardiovascular anesthesiologist showed a dilated left ventricle (LV), severe LV systolic dysfunction with an estimated ejection fraction (EF) of 20 to $25 \%$, and severe LV mid- and apical hypokinesis with preserved basal function (Videos 1 to 5). The 12-lead ECG obtained in the operating room $(\mathrm{OR})$ revealed sinus rhythm with no ischemic changes. Following extubation, she denied any symptoms consistent with acute coronary syndrome. On the 1st postoperative day, serum troponin peaked at $3.1 \mathrm{ng} / \mathrm{mL}$. A repeat TTE by cardiology showed a nondilated LV with moderate systolic dysfunction (calculated EF 31\%) and a similar pattern of mid- and distal LV wall motion abnormalities. Coronary angiography was performed revealing only nonocclusive disease ( $40 \%$ stenosis) of the mid-left anterior descending artery, establishing a presumptive diagnosis of takotsubo or stress cardiomyopathy. She required persistent vasopressor support due to hypotension for 2 days. Once hypotension resolved, metoprolol and lisinopril were started. After 12 days, she was discharged to a rehabilitation unit for 7 days and then home. The TTE prior to discharge was 
unchanged. The patient has not yet had her postdischarge follow-up appointment.

\section{Case 2}

A 55-year-old female with history of obesity, obstructive sleep apnea, hypertension, type II diabetes, smoking, and postlaminectomy pain syndrome with cervical and lumbar spinal cord stimulators presented for intrathecal morphine pump placement. Medications included methadone, oxycodone, metformin, pravastatin, and a multivitamin. She denied any previous history or current symptoms of ischemic heart disease or congestive heart failure. Chest radiograph was normal. The ECG showed normal sinus rhythm with left axis deviation. Anesthetic induction and surgery were uneventful. Following tracheal extubation, she developed severe dyspnea and hypoxemia. Following reintubation with auscultation of bilateral breath sounds and ventilation with $100 \%$ oxygen, oxygen saturations remained low. She was markedly hypertensive during this episode. Bronchoscopy confirmed satisfactory tracheal tube position and revealed pulmonary edema. Positive end expiratory pressure was increased and oxygenation improved. An ECG showed no ischemic changes. Transesophageal echocardiography (TEE) showed a dilated LV with moderately depressed LV global systolic function (EF 35\%), hypokinesis of the mid- and distal anterior, anteroseptal, and lateral walls, dyskinesis of the midposterior wall, and apical hyopkinesis (Videos 6 to 8). Serum troponin peaked at $0.46 \mathrm{mg} / \mathrm{mL}$ after 6 hours. The TTE on the day after surgery showed improvement in LV systolic function (EF $45-50 \%$ ), but persistent hypokinesis of the mid-LV segments circumferentially with preserved basal and apical function. Cardiology consultation was obtained and the patient was diagnosed with midventricular takotsubo. The patient was extubated on the 2 nd postoperative day and discharged home on 5 th postoperative day with follow-up including TTE in 3 months.

\section{Case 3}

A 48-year-old female with history of anxiety presented to the emergency department with a painful, cold, and discolored left lower extremity. She reported leg pain for several days and complete loss of motor function for about 24 hours. She also reported fatigue for several weeks, and recently had started taking escitalopram for anxiety surrounding psychosocial problems. Chest radiograph showed small bilateral pleural effusions, bibasilar opacities, and an enlarged cardiac silhouette all new compared with a prior study. The ECG showed sinus rhythm with anterolateral Q-waves not present on a historical ECG. Serum troponin was normal. A TTE ordered for syncope less than 1 year prior was normal. Vascular surgery was consulted and scheduled the patient for emergency lower extremity thrombectomy. On entry to the OR, heart rate was 125 beats/minute, blood pressure 135/95 $\mathrm{mm} \mathrm{Hg}$, and $\mathrm{SpO}_{2} 77 \%$. Because of her abnormal vital signs, chest X-ray, and ECG, the anesthesiologist performed focused TTE, which showed no pericardial effusion, a dilated LV with severe global LV systolic dysfunction (EF 25-30\%), and of the apical akinesis with possible apical thrombus (Video 9). As a result of these findings, the anesthetic plan was modified to include preinduction placement of a radial artery catheter for continuous hemodynamic monitoring, preparation of vasoactive infusions for immediate administration, and TEE to evaluate the suspected LV thrombus. After uneventful induction of general anesthesia, TEE confirmed the findings of LV apical akinesis with adherent thrombus, with mid-LV hypokinesis, and preserved basal function (Videos 10 and 11). Left superficial femoral artery and tibial artery thrombectomies and four-compartment fasciotomies were completed without incident. Postoperative cardiology consultation was obtained, and coronary angiography revealed no significant coronary artery disease. Unfortunately, below-the-knee amputation was subsequently required. Metoprolol, lisinopril, and enoxaparin were prescribed and the patient was discharged home after 3 weeks in hospital.

\section{DISCUSSION}

The differential diagnosis of abnormal LV regional wall motion includes several entities other than myocardial ischemia and infarction, summarized in Table $1 .^{1}$ Although the patients presented did not have prior echocardiograms available for comparison to definitively exclude preexisting cardiomyopathy or abnormal wall motion, their clinical presentations were temporally related to physiologic or psychological stress with a distribution of wall motion abnormalities unexplained by obstructive coronary artery disease, making takotsubo or stress cardiomyopathy the most likely diagnosis.

Table 1: Nonischemic wall motion abnormalities

Cardiac arrhythmias (atrial fibrillation, premature ventricular
contractions)
Hypovolemia
Pericardial constriction
Other types of cardiomyopathy (dilated cardiomyopathy from
viral illnesses, stress-induced cardiomyopathy)
Increased systemic afterload (aortic cross-clamping)
Left bundle branch block
Paced rhythms


Cases 1 and 3 exhibited the classic takotsubo pattern of apical and mid-LV hypokinesis in a noncoronary distribution, though other variants are seen as in case 2 .

While stress cardiomyopathy has been reported in a wide variety of perioperative settings, these cases highlight the role of anesthesiologist-performed TTE at the point of care in its prompt diagnosis and management. The pathophysiology, clinical presentation, and management of stress cardiomyopathy were recently covered in an excellent review by Weiner et al. ${ }^{2}$ In brief, stress cardiomyopathy is a form of nonischemic cardiomyopathy linked to endogenous or exogenous catecholamines characterized by profound and acute, but transient regional LV dysfunction (Table 2).

Focused echocardiography by anesthesia and critical care physicians rapidly adds valuable information in the search for causes of hemodynamic instability, shock, and hypoxemia, and can be used serially to evaluate the response to interventions. While TEE is firmly established as a modality for hemodynamic monitoring and therapy in cardiac and other high-risk surgeries, it is somewhat invasive and carries some risk of injury. Anesthesiologistperformed TTE is a newer concept with an advantage in terms of noninvasiveness, safety in patients with contraindications to TEE, and ability to be performed using highly portable systems in preoperative, intraoperative, and postoperative settings.

Intraoperative TTE is feasible, yielding at least two adequate quality imaging windows in $>90 \%$ of anesthetized patients positioned for various types of surgery. ${ }^{3}$ The same group further used focused TTE to examine 50 patients during intraoperative hemodynamic instability and found that TTE changed management in 33 patients $(66 \%) .{ }^{4} \mathrm{~A}$ recent systematic review examining the impact of focused TTE in noncardiac surgery and critical care found 18 publications (13 prospective and 5 retrospective), 13 of which reported a positive impact on patient management. ${ }^{5}$ The same group reported separately on the impact of echocardiography in the diagnosis and management of patients after cardiac surgery. ${ }^{6}$ Somewhat surprisingly, all seven publications reporting on focused TTE postcardiac surgery reported a high percentage of interpretable images, including both novice and expert users.

Table 2: Diagnostic criteria for stress-induced or takotsubo cardiomyopathy

\footnotetext{
Presence of a transient abnormality in left ventricular wall motion beyond a single coronary artery perfusion territory Absence of obstructive coronary artery disease or angiographic evidence of acute plaque rupture

New ECG abnormalities (ST elevation and/or T-wave inversion)

Modest elevation in troponin

Absence of myocarditis and pheochromocytoma
}

Preoperative echocardiography, according to the American Heart Association/American College of Cardiology guidelines, is not routinely indicated prior to noncardiac surgery, except for (1) evaluation of LV function in patients with symptoms of dyspnea or heart failure, and (2) for clinically suspected moderate or greater valvular disease if there has been no prior echocardiography within 1 year or there has been a change in clinical status. ${ }^{7}$ However, in certain populations, such as vascular surgery patients, it has been shown that the majority of patients with LV dysfunction are asymptomatic, but still at increased risk of adverse perioperative events. ${ }^{8} \mathrm{~A}$ prospective study of 99 patients presenting for emergency noncardiac surgery found that preoperative focused TTE screening changed the cardiac diagnosis compared with clinical examination in $67 \%$ of patients, and changed anesthesia management in $44 \% .{ }^{9}$ In another study of hip fracture surgery patients, preoperative focused TTE changed the cardiac diagnosis in $78 \%$ and the management plan in $52 \%$, with lower mortality in the focused TTE group compared with historical controls (not a controlled study). ${ }^{10}$ Sufficiently powered randomized controlled studies are needed to characterize populations in which preoperative screening TTE might improve outcomes. In the authors' practice, cardiovascular anesthesiologists with advanced echocardiography training perform preoperative limited TTE in selected patients undergoing elevated risk procedures (Table 3) and interface with the requesting physician to apply the findings within the specific clinical context. Results of focused TTE impact perioperative decision making by prompting a "step up" in patients with positive findings (e.g., delaying surgery for further evaluation or preoperative optimization, changes in anesthesia technique, escalation of monitoring, changes in drug therapy, such as inotropes/vasopressors, postoperative intensive care unit [ICU] admission), or prompting a "step down" in patients with normal exams (e.g., proceeding with surgery, avoiding unnecessary consultations and testing, and avoiding invasive monitoring and ICU admission). ${ }^{11}$ A systematic and goal-focused approach is used (Table 4).

Table 3: Possible indications for limited TTE in preoperative surgical patients

\footnotetext{
Patients with symptoms of cardiac disease, but never had cardiac evaluation or echocardiography

Patients with no symptoms, but inactive lifestyle and listed for major surgery

Patients older than 65 years of age

Patients with a heart murmur

Patients who had cardiac evaluation before, but there is a recent change in cardiac status

Patients with abnormal cardiac investigations, but no treatment or follow-up

Patients presenting for emergency/urgent surgery and cardiac status unknown
} 
Table 4: Approach to limited transthoracic preoperative evaluation

Left ventricular systolic function (qualitative, global, and segmental wall motion)

Right ventricular function (tricuspid annular plane motion and qualitative evaluation)

Mitral valve evaluation (qualitative mitral regurgitation evaluation, if mitral stenosis suspected, pressure gradients by continuous wave Doppler through mitral valve)

Aortic valve evaluation (aortic regurgitation evaluation and pressure gradients by continuous-wave Doppler in apical fivechamber or three-chamber views)

Tricuspid valve evaluation (tricuspid regurgitation evaluation and pulmonary artery systolic pressure quantification)

Volume status (qualitative left and right ventricular end-diastolic areas and quantitative inferior vena cava diameter and collapsibility) Left ventricular diastolic function (transmitral flow by pulse wave Doppler and mitral annular tissue Doppler)

Qualitative evaluation of atrial size

Inspection of aortic root and descending aorta

Pericardial and pleural effusion assessment

\section{CONCLUSION}

Perioperative echocardiography (both TEE and TTE) plays a pivotal role in the diagnosis and management of hemodynamic instability in critical care and noncardiac surgery. Increasing training in and use of focused TTE by anesthesiologists is a paradigm shift with potential to improve perioperative patient care and outcomes. Use of preoperative-focused TTE in high-risk patients is feasible and often changes anesthesia and monitoring strategies, potentially preventing critical events surrounding induction of anesthesia or intraoperatively and decreasing the necessity of TEE as "rescue" in the OR, though this concept needs further study.

\section{REFERENCES}

1. Citro R, Rigo F, Ciampi Q, D'Andrea A, Provenza G, Mirra M, Giudice R, Silvestri F, Di Benedetto G, Bossone E. Echocardiographic assessment of regional left ventricular wall motion abnormalities in patients with takotsubo cardiomyopathy: comparison with anterior myocardial infarction. Eur J Echocardiogr 2011 Jul;12(7):542-549.

2. Weiner MM, Asher DI, Augoustides JG, Evans AS, Patel PA, Gutsche JT, Mookadam F, Ramakrishna H. Takotsubo cardiomyopathy: a clinical update for the cardiovascular anesthesiologist. J Cardiothorac Vasc Anesth 2016 Jun 2. pii: S1053-0770(16)30174-4. [Epub ahead of print]

3. Kratz T, Campo dell'Orto M, Exner M, Timmesfeld N, Zoremba M, Wulf H, Steinfeldt T. Focused intraoperative transthoracic echocardiography by anesthesiologists: a feasibility study. Minerva Anestesiol 2015 May;81(5):490-496.

4. Kratz T, Steinfeldt T, Exner M, Dell Orto MC, Timmesfeld N, Kratz C, Skrodzki M, Wulf H, Zoremba M. Impact of focused intraoperative transthoracic echocardiography by anesthesiologists on management in hemodynamically unstable high-risk noncardiac surgery patients. J Cardiothorac Vasc Anesth 2016 Nov 2. pii: S1053-0770(16)30524-9. [Epub ahead of print].

5. Heiberg J, El-Ansary D, Canty DJ, Royse AG, Royse CF. Focused echocardiography: a systematic review of diagnostic and clinical decision-making in anaesthesia and critical care. Anaesthesia 2016 Sep;71(9):1091-1100.

6. Heiberg J, El-Ansary D, Royse CF, Royse AG, Alsaddique AA, Canty DJ. Transthoracic and transoesophageal echocardiography: a systematic review of feasibility and impact on diagnosis, management and outcome after cardiac surgery. Anaesthesia 2016 Oct;71(10):1210-1221.

7. Fleisher LA, Fleischmann KE, Auerbach AD, Barnason SA, Beckman JA, Bozkurt B, Davila-Roman VG, GerhardHerman MD, Holly TA, Kane GC. 2014 ACC/AHA guideline on perioperative cardiovascular evaluation and management of patients undergoing noncardiac surgery. J Am Coll Cardiol 2014 Dec;64(22):e77-e137.

8. Flu W-J, van Kuijk J-P, Hoeks SE, Kuiper R, Schouten O, Goei D, Elhendy A, Verhagen HJ, Thomson IR, Bax JJ. Prognostic implications of asymptomatic left ventricular dysfunction in patients undergoing vascular surgery. Anesthesiology 2010 Jun;112(6):1316-1324.

9. Canty DJ, Royse CF, Kilpatrick D, Williams DL, Royse AG. The impact of pre-operative focused transthoracic echocardiography in emergency non-cardiac surgery patients with known or risk of cardiac disease. Anaesthesia 2012 Jul;67(7): 714-720.

10. Canty DJ, Royse CF, Kilpatrick D, Bowyer A, Royse AG. The impact on cardiac diagnosis and mortality of focused transthoracic echocardiography in hip fracture surgery patients with increased risk of cardiac disease: a retrospective cohort study. Anaesthesia 2012 Nov;67(11):1202-1209.

11. Canty DJ, Royse CF, Kilpatrick D, Bowman L, Royse AG. The impact of focused transthoracic echocardiography in the pre-operative clinic. Anaesthesia 2012 Jun;67(6):618-625. 